\title{
Design of an Aperture-Coupled Microstrip Array Antenna for Millimeter-wave Radar System
}

\author{
Donghee Park ${ }^{1}$ and Yoonsik Kwak ${ }^{2}$ \\ ${ }^{I}$ Dept. of Information and Communications Engineering, Korea National Univ. of \\ Transportation, KNUT, \\ 50 Daehak-ro, Chungju-si, Republic of Korea \\ ${ }^{2}$ Dept. of Computer Engineering, Korea National Univ. of Transportation, KNUT, \\ 50 Daehak-ro, Chungju-si, Republic of Korea (Corresponding Author) \\ \{dhpark,yskwak\}@ut.ac.kr
}

\begin{abstract}
In this paper, we designed and interpreted the array antennas of the aperture-coupled microstrip operating in the millimeter-wave band for radar system. The microstrip array antenna can be designed with a transmission line circuit and a matching circuit in the same substrate. Therefore it is easy to combine with the active elements of the system. We add the slots to the patch to overcome it because general microstrip patch antennas have narrow band characteristics. we have designed the $U$-shaped slots in the patch. The substrate of patch for an aperture-coupled is used for general dielectric layer, and the substrate of transmission line for T-junction feeding is used FR4-epoxy. At this time, the analyzed center frequencies are 17 resonance mode from $24 \mathrm{GHz}$ to $77 \mathrm{GHz}$ at the $4 \times 4$ array. As a result, this paper is proposed the possibility of prototyping by design of multi-mode array antenna in the millimeter-wave.
\end{abstract}

Keywords: Aperture-coupled antenna, millimeter-wave antenna, multi-band antenna, patch slots antenna, patch array antenna

\section{Introduction}

The study on millimeter-wave antennas have evolved continuously over the past 30 years, with the rapid development of microstrip antenna theories and techniques.

The microstrip antenna has been applied to many fields because it can be integrated a small weight and volume. In recent years, millimeter-wave radar systems in the transport sector are being used widely in the automotive electronics sensors with the information and communication technologies.

In the center of these changes, it is an Adaptive Cruise Control (ACC) system. The system is an active safety device that can predict the occurrence of an accident by sensing the external environment while driving car [1]-[3].

In recently, the development of automotive radar systems divided by a short distance SRR (Short Range Radar) and long distance LRR (Long Range Radar) are being developed. The SRR was used UWB of $24 \mathrm{GHz}$ band and $79 \mathrm{GHz}$ band, the LRR has been used for the 77GHz-band.

Future of Automotive radar systems will be integrated into one radar system SRR and LRR. FMCW modulation is expected to be widely used in the integrated system [4].

This paper is designed and interpreted microstrip array antenna(MAA) of the aperturecoupled microstrip antennas operating in the millimeter wave band. In this technique the feed network is separated from the radiating patch by a common ground plane. Energy is electromagnetically coupled through an aperture in the ground. This aperture is usually centered with respect to the patch where the patch has its maximum magnetic field. 
The MAA can be designed with a transmission line circuit and a matching circuit in the same substrate. Therefore it is easy to combine with the active elements of the system. Its main advantage is that it can be an overall design of the semiconductor integrated circuit.

We add the slots to the patch to overcome it because general microstrip patch antennas have narrow band characteristics. Also, it can be used to achieve dual or multiple frequency operation. The shape of

slot selects a symmetric structure of the U-shape with a multi-band. As a result, this paper presents the possibility of a multi-band array antenna system implementations through the simulation results.

\section{Aperture-Coupled Microstrip Single Patch Antenna}

The aperture-coupled microstrip single patch antenna used in this study is shown in Figure 1. This technique has several advantages, which makes it suitable for widespread applications in communication systems. The feed substrate is usually thin with high permittivity, whereas the patch substrate can be thick with low permittivity. In this configuration the antenna system is composed of a conductive thin metal plate and an air layer to minimize the reflected wave, the feed line for supplying a signal, the dielectric layer for supporting a surface, a conductive ground plane that contains the aperture, the supporting dielectric layer physically and the patch $[5,6]$.

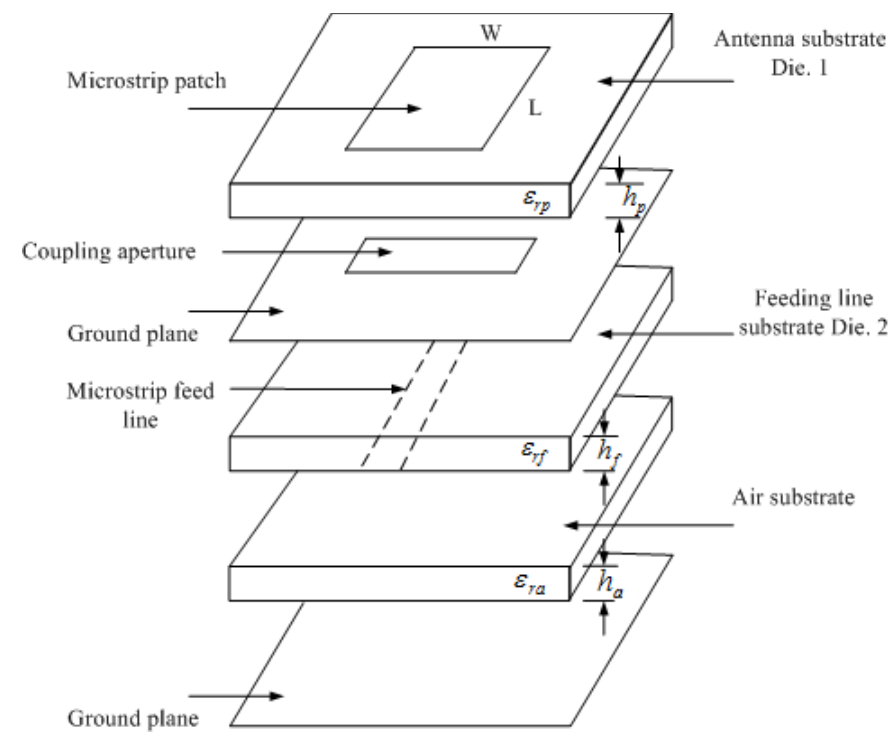

Figure 1. Aperture-coupled Microstrip Antenna

In general, the size of the patch is determined below half-wave by the fundamental resonance mode. In this case, the selected resonant frequency is $24 \mathrm{GHz}$. Therefore, practical length $\mathrm{L}$ and width $\mathrm{W}$ of the patch are obtained as follows.

$$
\begin{aligned}
& L=\frac{c}{2 f_{\tau} \sqrt{\epsilon_{r \varepsilon t f}}}-2 \Delta L \\
& W=\frac{c}{2 f_{\tau}}\left(\frac{\epsilon_{r y}+1}{2}\right)^{-1 / 2}
\end{aligned}
$$

In this equation (1), greff is the effective dielectric constant of the substrate considering the edge effect, fr is a resonance frequency of the antenna, c is the speed of light in free space region, erp is the relative dielectric constant, and $\Delta \mathrm{L}$ represents the equivalent length of the patch according to the size of the operating frequency. 
The value $\Delta \mathrm{L}$ is determined from the following equation. The width $\mathrm{W}$ of the patch is mainly used to obtain the input impedance.

$$
\Delta L=0.412 h \frac{\left(\epsilon_{r s f f}+0.3\right)(W / h+0.264)}{\left(\epsilon_{r s t f}-0.258\right)(W / h+0.8)}
$$

The dielectric substrate Die. 1 is Rogers RT / duroid 5880 and it have the relative dielectric constant $\operatorname{crp}=2.2$, the loss tangent $\tan \delta=0.0009$, and the thickness of the substrate $\mathrm{hp}=0.3$ $\mathrm{mm}$.

As shown in Figure 1, the substrate Die. 2 for a microstrip line is FR-4epoxy and it is the relative dielectric constant $\operatorname{crf}=3.92$, the loss tangent $\tan \delta=0.031$, and a thickness $\mathrm{hf}=0.5$ $\mathrm{mm}$. Also the air layer with a thickness ha $=1.6 \mathrm{~mm}$ to minimize the radiation of the strip line is added.

The final size of the patch is designed to have the width $\mathrm{W}=6 \mathrm{~mm}$ and length $\mathrm{L}=4 \mathrm{~mm}$. In addition, the width of the aperture coupled microstrip line with a $50 \Omega$ characteristic is W01 $=0.4 \mathrm{~mm}$. The width of the slot for aperture coupled inside ground plane is $S \mathrm{w}=0.1 \mathrm{~mm}$ and length is $\mathrm{SL}=4.5 \mathrm{~mm}$.

This paper is added to the slot in the patch have a multi-band characteristics within the millimeter-wave band. In particular, the shape of the slot in the patch is selected by a Ushaped slot to have a multi-band characteristics from K-band to W-band[7]-[9]. The shape of the slot is shown in Figure 2. Here, the width of the slot is $\mathrm{Ws}=0.5 \mathrm{~mm}$, the length of the slot $\mathrm{d} 1=1.8 \mathrm{~mm}$, and the height of the slot is $\mathrm{d} 2=3.2 \mathrm{~mm}$ respectively.

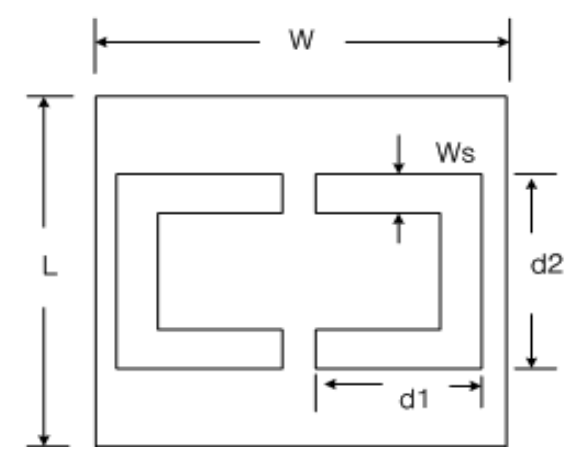

Figure 2. Single Patch Antenna with U-shaped Slots

\section{Aperture-Coupled Antenna Arrays}

The antenna arrays are designed starting with a single element using the transmission line model described in Section II. Figure 3 shows the position of the feed structures and the elements of a $4 \times 4$ antenna array. This antenna is designed with microstrip line feed network employing a T-junction. Each reactive T-junction used in the antenna feed network provides and equal or $3 \mathrm{~dB}$ power split. The width Wo1 of the characteristic impedance $50 \mathrm{Ohm}$ is 0.4 $\mathrm{mm}$, and the width Wo2 of the $70.7 \mathrm{Ohm}$ line is $0.3 \mathrm{~mm}$. The interval Dx and Dy between the patch and the patch are $5.4 \mathrm{~mm}$. 


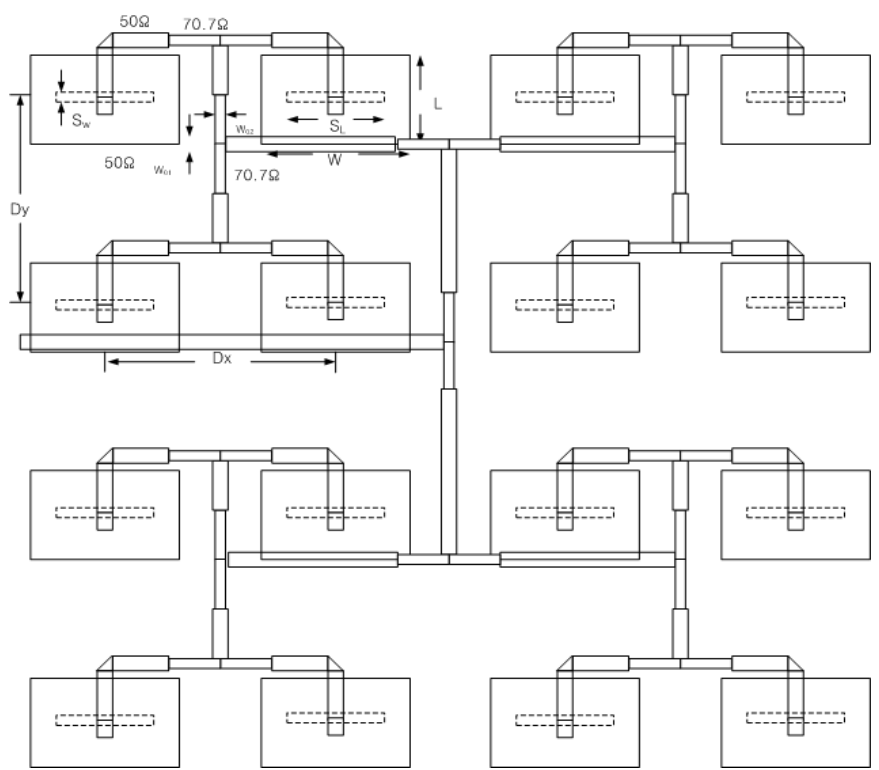

Figure 3. Layout of $4 \times 4$ Array with T-junction Dividers

\section{Analysis of Multi-band Array Antenna}

The return loss of a single patch, $2 \times 1,2 \times 2,4 \times 2$, and $4 \times 4$ aperture coupled array with feed network using T-junction divider are displayed in Figure 4. The number of resonantband in single patch is $3,2 \times 1$ array is $8,2 \times 2$ array is $10,4 \times 2$ array is 14 , and $4 \times 4$ array is 17.

The resonant frequency of the multi-mode is selected to adjust finely the length of the stub. In $4 \times 4$ array antenna, we are selected the length of stub as $0.5 \mathrm{~mm}$ to obtain the maximum number of resonance mode. The proposed geometry is simulated using HFSS tool software.

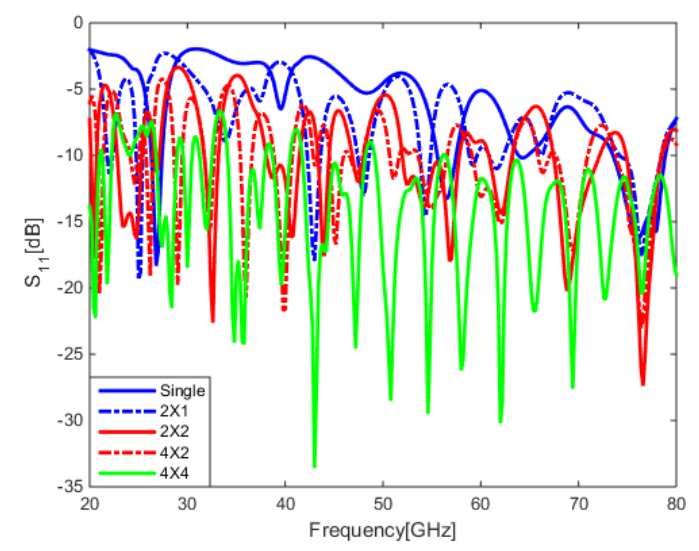

Figure 4. The Return Loss Curves for Single Patch, $2 \times 1$ Array, $2 \times 2$ Array, $4 \times 2$ Array, and $4 \times 4$ Array

Figure 5 presents the results of the radiation pattern at the center frequency of $24.4 \mathrm{GHz}$ and $77.6 \mathrm{GHz}$ of the third-bands of a single patch antenna. Figure 6 presents the results of the radiation pattern at $25 \mathrm{GHz}$ and $76 \mathrm{GHz}$ of 8 - band of $2 \times 1$ array antenna. Figure 7 presents the results of the radiation pattern at $24 \mathrm{GHz}$ and $77 \mathrm{GHz}$ of 10 - band of $2 \times 2$ array antenna. Figure 8 presents the results of the radiation pattern at $24 \mathrm{GHz}$ and $77 \mathrm{GHz}$ of 14 - band of $4 \times 2$ array antenna. Figure 9 presents the results of the radiation pattern at $24 \mathrm{GHz}$ and 77 $\mathrm{GHz}$ of 17 - band of $4 \times 4$ array antenna. 


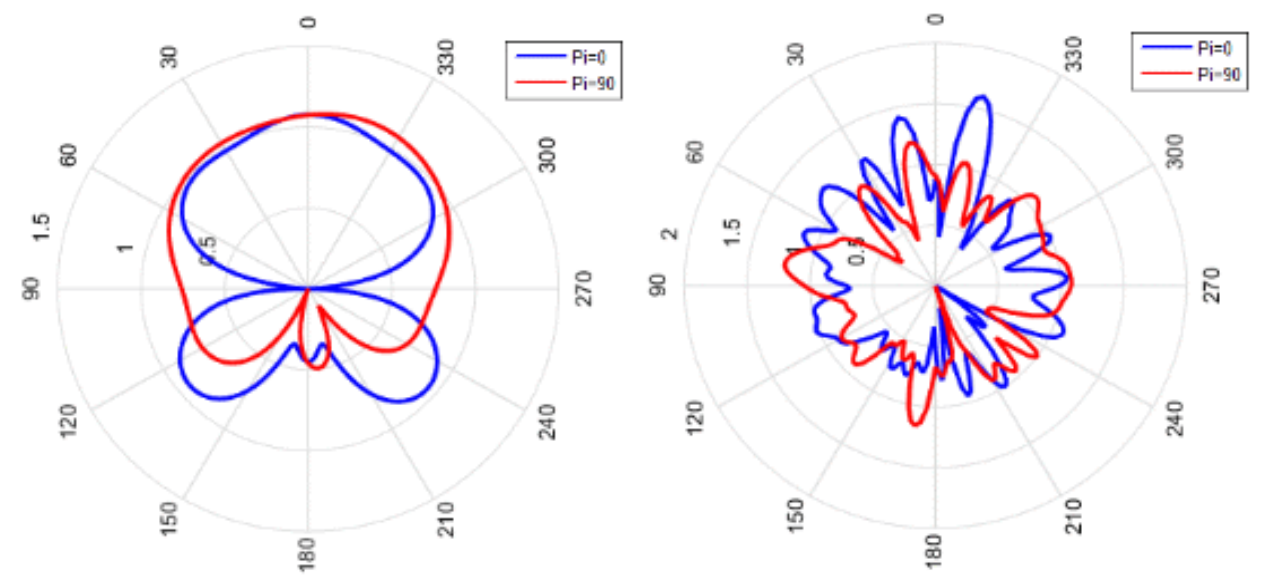

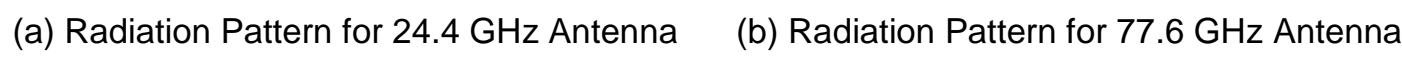

Figure 5. The Radiation Patterns of Single Patch Antenna

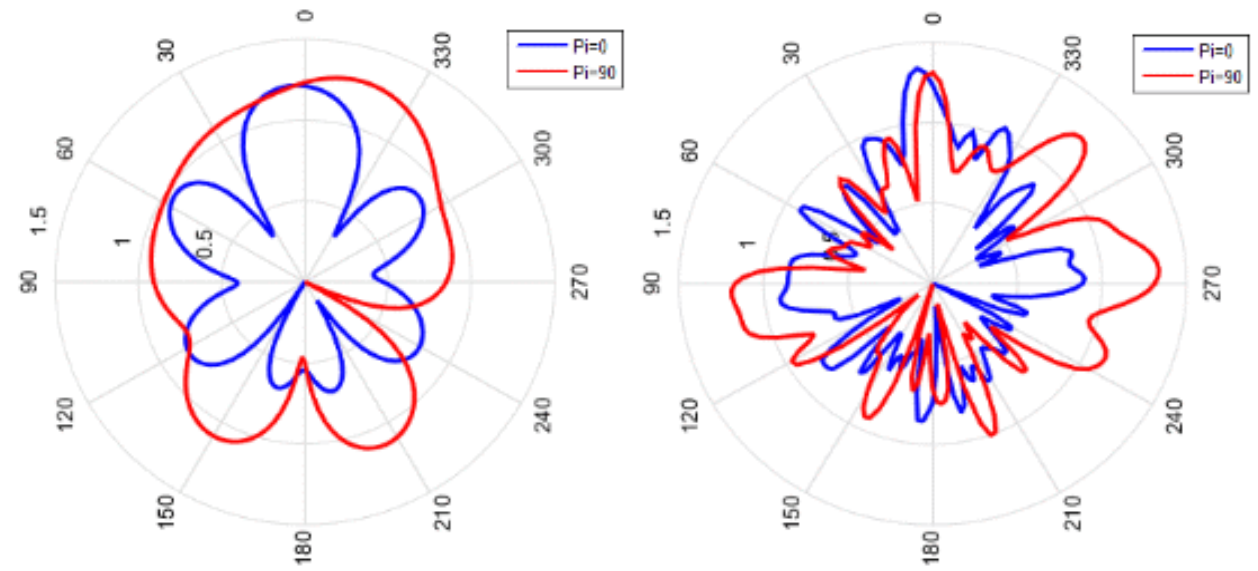

(a) Radiation Pattern for $25 \mathrm{GHz}$ Antenna $\quad$ (b) Radiation Pattern for $76 \mathrm{GHz}$ Antenna

Figure 6. The Radiation Patterns of $2 \times 1$ Array Antenna

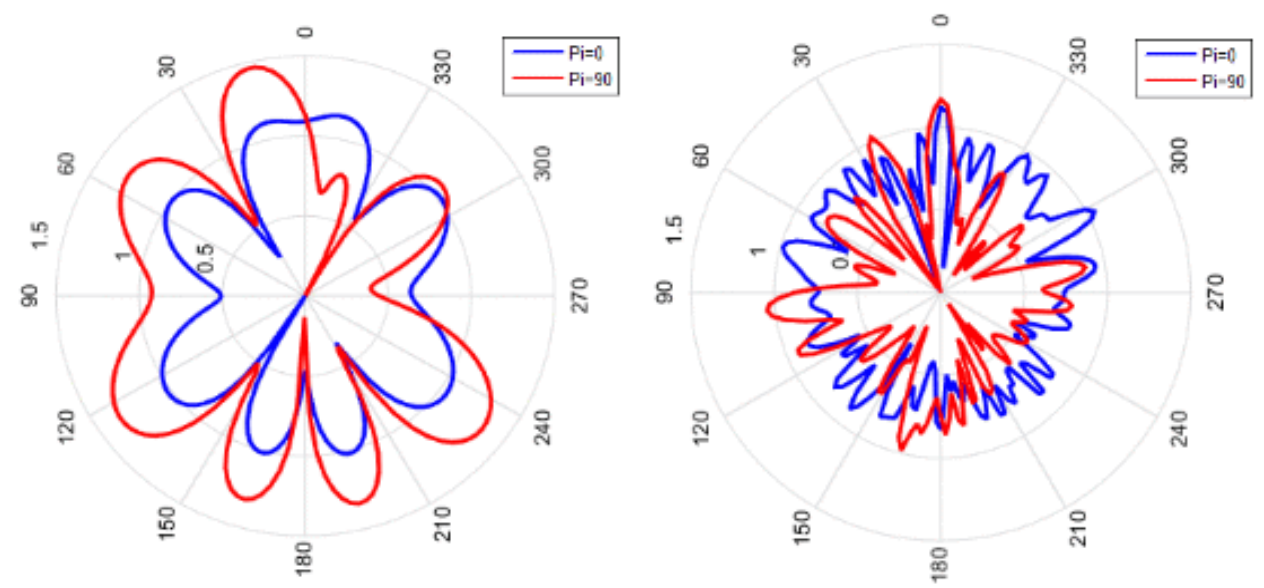

(a) Radiation Pattern for $24 \mathrm{GHz}$ Antenna (b) Radiation Pattern for $77 \mathrm{GHz}$ Antenna

Figure 7. The Radiation Patterns of $2 \times 2$ Array Antenna 


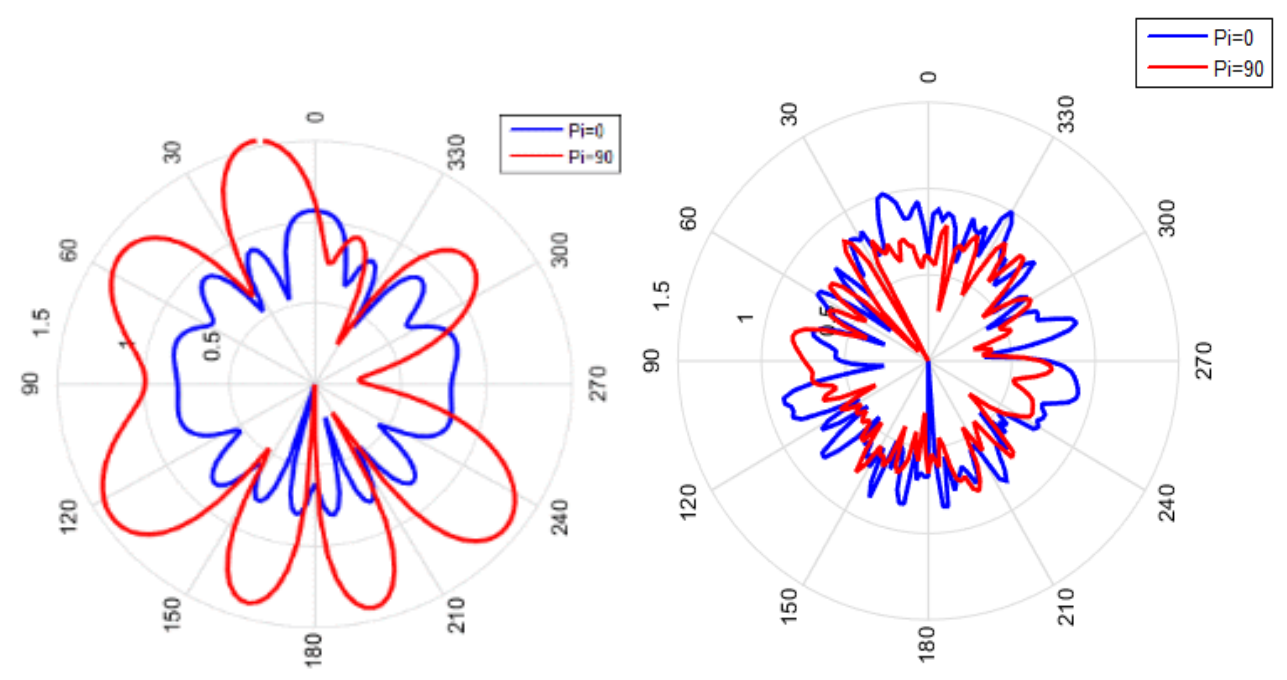

(a) Radiation Pattern for $24 \mathrm{GHz}$ Antenna (b) Radiation Pattern for $77 \mathrm{GHz}$ Antenna

Figure 8. The Radiation Patterns of $4 \times 2$ Array Antenna
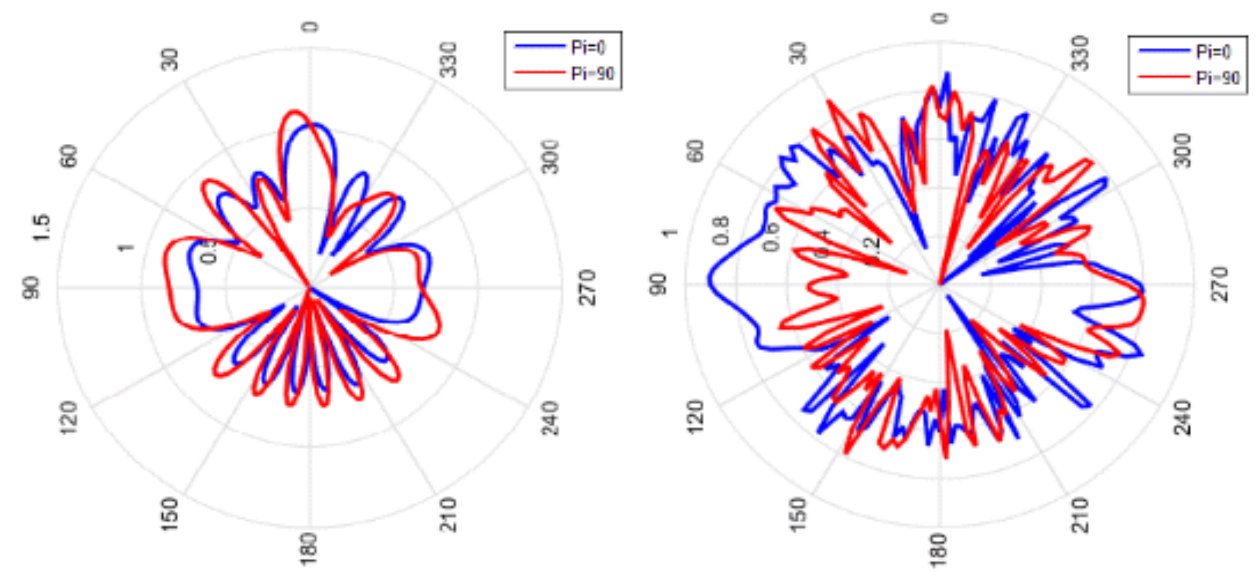

(a) Radiation Pattern for $24 \mathrm{GHz}$ Antenna (b) Radiation Pattern for $77 \mathrm{GHz}$ Antenna

Figure 9. The Radiation Patterns of $4 \times 4$ Array Antenna

\section{Conclusion}

In this paper, we designed and interpreted the MAA of the aperture-coupled microstrip antennas operating in the millimeter-wave band for radar system. The MAA can be designed with a transmission line circuit and a matching circuit in the same substrate. Therefore it is easy to combine with the active elements of the system.

We add the slots to the patch to overcome it because general microstrip patch antennas have narrow band characteristics. Also, it can be used to achieve dual or multiple frequency operation.

The return loss of a single patch, $2 \times 1,2 \times_{2}, 4 \times_{2}$, and $4 \times 4$ aperture coupled array with feed network using T-junction divider are analyzed here. The number of resonant-band in single patch is $3,2 \times 1$ array is $8,2 \times 2$ array is $10,4 \times 2$ array is 14 , and $4 \times 4$ array is 17 .

As a result, this paper is available to have multiple resonant mode in the millimeter-wave band. Also, this paper is required to extend over the $4 \times 4$ array antenna in millimeter-wave band. 


\section{References}

[1] D. M. Pozar, "Considerations for Millimeter Wave Printed Antennas," IEEE Trans. Antennas Propagat., vol. 31, no. 5, (1983), pp.740-747.

[2] C. A. Balanis, Antenna Theory Analysis and Design, $2^{\text {nd }}$ ed., New York: John Wiley \& Sons, (1997).

[3] M. Stotz, G. Gottwald, H. Haspeklo, and J. Wenger, "Planar Millimeter-Wave Antennas Using SiNx-Membranes on GaAs", IEEE Trans. Microwave Theory Tech., vol. 44, no. 9, (1996), pp.1593-1595.

[4] M. Slovic, B. Jokanovic and Kolundzija, "High efficiency patch antenna for $24 \mathrm{GHz}$ anticollision radar", Telecommunications in Modern Satellite, Cable and Broadcasting Services, $7^{\text {th }}$ International Conference, vol. 1, (2005), pp. 20-23.

[5] S. C. Shrivastava and Z. Aijaz, "An Intriduction of Aperture Coupled Microstrip Slot Antenna", International Journal of Engineering and Technology, vol. 2, no. 1, (2010), pp. 36-39.

[6] K. Pitra, and Z. Raida, "Planar Millimeter-Wave Antennas: A Comparative Study", Radio Engineering, vol. 20, no. 1, (2011), pp. 263-269.

[7] P. Pursula, T. Vähä-Heikkilä, A. Muller, G. Konstantinidis, D. Neculoiu, A. Oja, and J. Tuovinen, "Millimeter wave identification-New radio system for low power, high data rate and short range", IEEE Trans. Microw. Theory Tech., to be published.

[8] A. Babakhani, X. Guan, A. Komijani, A. Natarajan, and A. Hajimiri, "A 77-GHz phased-array transceiver with on-chip antennas in silicon", IEEE J. Solid-State Circuits, vol. 41, no. 12, (2006), pp. 2795-2806.

[9] Y. P. Zhang, M. Sun, and L. H. Guo, "On-chip antennas for 60-GHz radios in silicon technology", IEEE Trans. Electron Devices, vol. 52, no. 7, (2005), pp. 1664-1668.

\section{Authors}

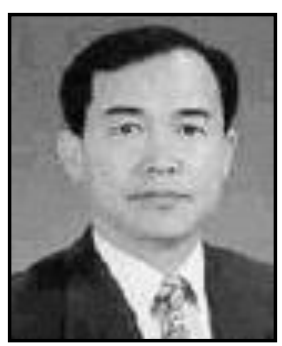

Donghee Park, he received the B.S. degree in electrical engineering from the University of Cheongju in 1985, and the M.S. and Ph.D. degrees in electrical engineering from the University of Chung-Ang in 1992. In 1992, he joined the Department of Information and Communications Engineering, Korea National University of Transportation, Chungju, Korea, where he is now a Professor. His research interests are electromagnetic wave theory, the design and analysis of microwave, millimeter wave, terahertz wave, and nano-structured antennas.

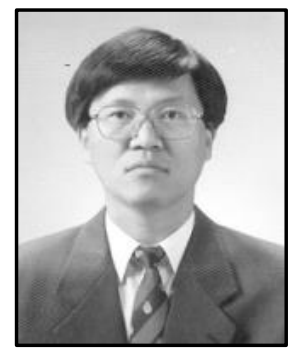

Yoonsik Kwak, he received his B.S. degree in Electrical Engineering from the University of Cheongju in 1984, his M.S.E.E. degree from the University of Kyunghee in 1986 and his Ph.D. degree from the University of Kyunghee in 1994. He worked at Korea National University of Transportation in the Department of Computer Engineering and rose to the level of Full Professor. His research interests are in the areas of signal processing, Internet communication, microcomputer system, and applications of these methods to mobile system. 
International Journal of Control and Automation Vol. 8, No. 1 (2015) 